\title{
The Focusing Problem for the Eikonal Equation
}

\author{
S. B.Angenent and D. G.Aronson
}

Dedicated to the Memory of Philippe Bénilan

\begin{abstract}
We study the focusing problem for the eikonal equation$$
\partial_{t} u=|\nabla u|^{2}
$$

i.e., the initial value problem in which the support of the initial datum is outside some compact set in $\mathbf{R}^{d}$. The hole in the support will be filled in finite time and we are interested in the asymptotics of the hole as it closes. We show that in the radially symmetric case there are self-similar asymptotics, while in the absence of radial symmetry essentially any convex final shape is possible. However, for generic initial data the asymptotic shape will be either a vanishing triangle or the region between two parabolas moving in opposite directions (a closing eye). We compare these results with the known results for the porous medium pressure equation which approaches the eikonal equation in the limit as $m \rightarrow 1$.
\end{abstract}

\section{Introduction}

In this paper we compare the focusing problem for the eikonal equation

$$
\partial_{t} u=|\nabla u|^{2}
$$

with the corresponding problem for the porous medium (pressure) equation

$$
\partial_{t} u=(m-1) u \Delta u+|\nabla u|^{2},
$$

in $\mathbb{R}^{d} \times \mathbb{R}^{+}$for $d \geq 1$. Here $\nabla$ denotes the gradient with respect to the spatial variables $\left(x_{1}, x_{2}, \cdots, x_{d}\right)$. The quantity $m>1$ in (PME) is a constant.

Consider the initial value problem for either (EE) or (PME) with the initial datum $u(x, 0)=u_{0}(x)$. We assume that $u_{0}$ is compactly supported and has a "regular free boundary", i.e.,

$$
u_{0}(x)=\max \left(\widetilde{u}_{0}(x), 0\right)
$$

for some $\widetilde{u}_{0} \in C^{\infty}\left(\mathbf{R}^{d}\right)$, where 0 is a regular value of $\widetilde{u}_{0}$ and $\left\{x \mid \widetilde{u}_{0}(x) \geq 0\right\}$ is compact. The viscosity solution to the initial value problem for (EE) with initial datum $u(x, 0)=u_{0}(x)$ is a continuous function of $(x, t)$ (cf. [17]). The same is true for the generalized solution to the corresponding initial value problem for (PME) (cf. [8]). The zero set

$$
Z_{t}=\left\{x \in \mathbf{R}^{d}: u(x, t)=0\right\}
$$


of either the viscosity or generalized solution has one unbounded component. Let $K_{t}$ denote the union of all bounded components of $Z_{t}$. For the focusing problem we assume that $K_{0}$ is a compact simply connected set, i.e., that the support of $u_{0}$ "has a hole." The sets $K_{t}$ form a nonincreasing and eventually strictly decreasing family of subsets of $\mathbf{R}^{d}$ which will become empty at some finite time $T$

$$
T=\inf \left\{t: K_{t}=\varnothing\right\}
$$

is called the focusing time and we say that the solution has focused (or filled the hole $K_{0}$ ) at time $t=T$.

The eikonal equation (EE) shows up as a formal limit of (PME) as $m \searrow 1$, and indeed, it has been shown in [17] that weak solutions to the initial value problem for (PME) converge uniformly as $m \searrow 1$ to viscosity solutions of the corresponding problem for (EE). The absence of diffusion makes the eikonal equation considerably simpler than (PME), and in fact there is even an explicit formula for the viscosity solution in terms of its initial data,

$$
u(x, t)=\sup _{y \in \mathbb{R}^{d}}\left\{u_{0}(y)-\frac{|x-y|^{2}}{4 t}\right\} .
$$

This representation of the solution is called the Lax-Hopfformula. See [17] or [15] for a discussion. From this formula for the viscosity solution one immediately deduces the following representation of the free boundary. The free boundary is the graph in spacetime of the filling time

$$
T_{*}(x)=\inf _{u(x, t)>0} t=\inf _{u_{0}(y)>0} \frac{|x-y|^{2}}{4 u_{0}(y)} .
$$

The Lax-Hopf formula and the formula (2) for the filling time allow us to study focusing of solutions to (EE) in much more detail than can be done at present for (PME). In this note we compare the results on focusing for the eikonal equation with what is known for (PME).

In the non-generic case of radial symmetry, $K_{t}$ is, of course, a $d$-dimensional ball. We show that in this case the viscosity solution $u$ for (EE) behaves like a self-similar solution near focusing. More precisely, we show that

$$
\lim _{\varepsilon \searrow 0} \frac{1}{\varepsilon} u(\varepsilon \xi, T+\varepsilon \tau)=c|\xi|+c^{2} \tau
$$

uniformly for bounded $\xi \in \mathbb{R}^{d}$ and bounded $\tau \in \mathbb{R}$. This extends our results [1] for (PME) to the case $m=1$. In [1] it was shown for any $1<m<\infty$ that for a radially symmetric weak solution of (PME) which focusses at $t=T$ one has

$$
\lim _{\varepsilon \searrow 0} \varepsilon^{\alpha-2} u\left(\varepsilon \xi, T-\varepsilon^{\alpha} \tau\right)=c^{2} V_{m}\left(\frac{|\xi|}{c \tau^{1 / \alpha}}\right),
$$

in which $V_{m}$ is the Aronson-Graveleau profile, $\alpha \in(1,2)$ is the corresponding exponent, and $0<c<\infty$ is a positive constant depending on the initial data.

In the nonradial case we show that the eikonal equation admits many more self-similar focusing solutions than PME. In particular we show that for any closed convex set $C \subset$ 
$\mathbb{R}^{d}$ containing the origin there is a self-similar viscosity solution $S(x, t)$ (i.e. one which satisfies $S(\lambda x, \lambda t)=\lambda S(x, t)$ for all $\lambda>0)$ such that

$$
\left\{x \in \mathbb{R}^{d} \mid S(x, t)=0\right\}=(-t) C
$$

for all $t<0$. In other words, for the eikonal equation "a vanishing hole can have any convex shape."

This contrasts sharply with the porous medium equation. All self-similar focusing solutions for PME which have been constructed either analytically [2] or numerically [3] have some kind of discrete symmetry. Moreover, in [2] it is shown that self-similar focusing solutions satisfy a nonlinear elliptic free boundary problem which can be transformed to a nonlinear Fredholm equation. One therefore expects self-similar focusing solutions to PME to occur in discrete families, and not in infinite dimensional continua as is the case for the eikonal equation.

We next consider small perturbations of radially symmetric initial data. We show that, to leading order, $\partial K_{t}$ propagates according to Huygen's principle, i.e., with constant normal velocity. More precisely, if $u^{0}(x, t)=U(|x|, t)$ is a radially symmetric solution which focusses at time $t=T$, then we observe that the viscosity solution $u^{\varepsilon}$ with initial data $u^{\varepsilon}(x, 0)=U(|x|)+\varepsilon g(x)$ satisfies

$$
\lim _{\varepsilon \rightarrow 0} \frac{1}{\varepsilon} u^{\varepsilon}(\varepsilon \xi, T+\varepsilon \tau)=V(\xi, \tau)
$$

where $V$ is a viscosity solution of the Huygens-Hamilton-Jacobi equation

$$
\partial_{\tau} V=c|\nabla V| \text {. }
$$

The level sets of viscosity solutions to this equation are fronts which evolve by Huygens' principle, i.e. they propagate with constant normal velocity $c$. Moreover, we observe that any solution of (5) can occur as the limit in (4), provided $|\nabla V| \equiv c$.

This implies that radial focusing is unstable: a small perturbation will turn a radial focusing solution into one of the two generic focusing solutions described below. This instability also appears to be present in (PME). For (PME) radial holefilling is described by the Aronson-Graveleau profile, but if one perturbs a radial solution slightly, then numerical computations indicate that the perturbations will grow, and that the solution will generally not return to radial symmetry. However, for (PME) one expects a sufficiently symmetric peturbation to die out and disappear, at least if $m$ is large enough. The instability of radial focusing for (EE) combined with Lions-Souganidis-Vázquez' convergence result [17] gives a heuristic reason for the occurrence of the infinite sequence of bifurcations found in [2]: for large values of $m$ the diffusion term in (PME) is dominant, and the radial selfsimilar solution will only have a small number of unstable modes, but for $m=1+o(1)$ the solution to (PME) tries to follow the viscosity solution to (EE), where radial focussing has infinitely many unstable modes.

Finally, we consider generic solutions. For generic initial data, i.e. for an open and dense set of $\tilde{u}_{0} \in C^{\infty}\left(\mathbb{R}^{d}\right), K_{t}$ shrinks to a point and, when $d=2$, we can characterize the generic possibilities for the final form of $\partial K_{t}$. These are either 

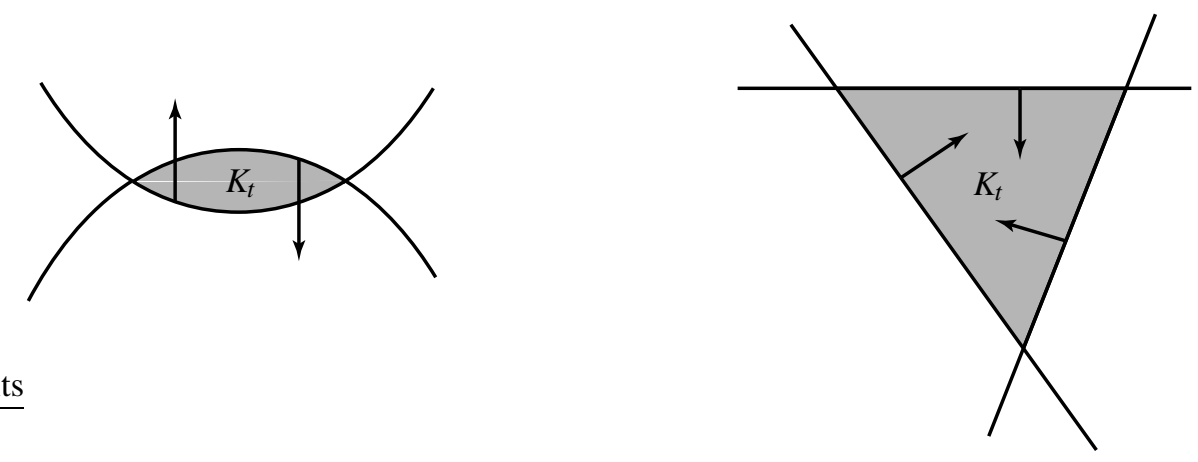

FIGURE 1. The closing eye and vanishing triangle solutions

A Closing Eye. $K_{t}$ approximates the region between two parabolae

$$
y= \pm\left\{c_{ \pm}(T-t)-x^{2}\right\} .
$$

The width of $K_{t}$ is $O(\sqrt{T-t})$ while its height is $O(T-t)$. The aspect ratio of $K_{t}$ tends to infinity as $t \nearrow T$ (cf. Figure 1).

A Vanishing Triangle. $K_{t}$ is approximately the region enclosed by three straight lines which propagate with constant normal velocity, and which pass through a common point at $t=T$ (cf. Figure 1)

Numerics and asymptotic analysis strongly suggest that there are solutions to (PME) close to the closing eye $([\mathbf{3}, \mathbf{1 1}])$ for any $m>1$, even though there is as yet no rigorous theory.

The situation with the vanishing triangle is different: numerics again suggest that there exists a self-similar solution whose free boundary approximates a triangle when $m-1$ is small enough, but this triangle must be an equilateral triangle. Thus it seems that the second kind of generic hole filling for (EE) is highly nongeneric for (PME). Again, rigorous proofs for the statements about (PME) are lacking.

Acknowledgements. The authors would like to thank Ilya Bogaevsky for providing the (p)reprints [12], [13], [14] and his comments on an earlier version of this paper. The authors also would like to thank Craig Evans for some helpful elementary remarks on viscosity solutions.

\section{Radial Solutions}

If the initial function $u_{0}(x)$ is radially symmetric, i.e. $u_{0}(x)=U_{0}(r), r=|x|$, then the viscosity solution $u(t, x)$ will be radially symmetric for all $t>0$. Indeed, the Lax-Hopf formula reduces to

$$
U(t, r)=\sup _{\rho>0}\left\{U_{0}(\rho)-\frac{(r-\rho)^{2}}{4 t}\right\} .
$$

This equation is obtained from (1) by maximizing over all $y$ with $|y|=\rho$. So we see that the radial solution is exactly the same as the viscosity solution in one space dimension. 
This makes it easy to understand generic radial hole filling. Consider an initial function $U_{0}(r)$ as in Figure 2.

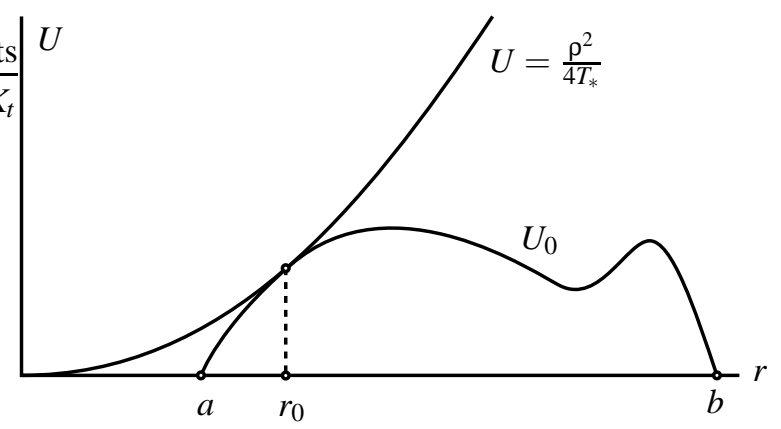

FIgURE 2. Computing the Radial Hole Filling Time

The unique hole in the center will fill up in time

$$
T_{*}=\sup _{\rho>0, U_{0}(\rho)>0} \frac{\rho^{2}}{4 U_{0}(\rho)} .
$$

Let us assume that the function $\rho \mapsto \rho^{2} / 4 U_{0}(\rho)$ has a unique maximum at $\rho=r_{0}$ as will be the case for a generic smooth initial $U_{0}$. Then, at the hole filling time $T_{*}$ the solution near $r=0$ will be a smooth function of $r$, and if we allow $r<0$ in (6), then it extends to a smooth function in a full neighborhood in $\mathbb{R}^{2}$ of $\left(r=0, t=T_{*}\right)$. At $r=0, t=T_{*}$ the method of characteristics tells us that $U_{r}=U^{\prime}\left(r_{0}\right)$; the eikonal equation enforces $U_{t}\left(0, T_{*}\right)=\left(U_{r}\right)^{2}=$ $U^{\prime}\left(r_{0}\right)^{2}$. Thus, locally we have

$$
U(r, t)=U^{\prime}\left(r_{0}\right) r+U^{\prime}\left(r_{0}\right)^{2}\left(t-T_{*}\right)+O\left(r^{2}+\left(t-T_{*}\right)^{2}\right) .
$$

Hence

$$
\lim _{\varepsilon \searrow 0} \frac{1}{\varepsilon} U\left(\varepsilon R, T_{*}+\varepsilon \tau\right)=U^{\prime}\left(r_{0}\right) R+U^{\prime}\left(r_{0}\right)^{2} \tau .
$$

As we noted in the Introduction, this is analogous to the radial hole-filling asymptotics for the porous medium equation. Here we see that that radial hole filling is described by a self-similar solution

$$
U_{c}(r, t)=c(r+c t)_{+},
$$

and that these self-similar solutions occur in a one parameter family. For the porous medium equation the self-similar solutions are the Aronson-Graveleau solutions, for the eikonal equation they are simply plane waves converging upon the origin. 


\section{Nonradial Self-Similar Solutions}

For any vector $\xi \in \mathbb{R}^{d}$ the plane wave solution

$$
W_{\xi}(x, t)=\left(\xi \cdot x+|\xi|^{2} t\right)_{+}
$$

is a self-similar solution which reaches the origin at $t=0$.

LEMMA 1. For any closed subset $F \subset \mathbb{R}^{d}$ the function

$$
S_{F}(x, t)=\sup _{\xi \in F} W_{\xi}(x, t)
$$

is a self-similar solution which reaches the origin at time $t=0$.

The "hole" in support of the self-similar solutions constructed here is the set

$$
\begin{aligned}
K_{F}(t) & =\left\{x \in \mathbb{R}^{d} \mid S_{F}(x, t)=0\right\} \\
& =\left\{\left.x \in \mathbb{R}^{d}|\forall \xi \in F \xi \cdot x+| \xi\right|^{2} t \leq 0\right\} \\
& =\left\{x \in \mathbb{R}^{d}\left|\forall \xi \in F \frac{\xi}{|\xi|} \cdot x+\right| \xi \mid t \leq 0\right\} \\
& =\bigcap_{\xi \in F}\left\{x\left|\frac{\xi}{|\xi|} \cdot x \leq-\right| \xi \mid t\right\} .
\end{aligned}
$$

Thus we see that for $t<0$ the hole $K_{F}(t)$ is a nonempty convex set whose support function $p: S^{d-1} \rightarrow \mathbb{R}$ is given by

$$
p(\omega)=\inf \{p>0 \mid-t p \omega \in F\}
$$

Furthermore, any compact convex set $C \subset \mathbb{R}^{d}$ containing the origin can be represented as $C=K_{F}(-1)$, with $K_{F}(-1)$ defined as in (8) for some suitably chosen $F \subset \mathbb{R}^{d}$. Therefore there are uncountably many self similar solutions, and that self-similarity of a solution to the eikonal equation does not guarantee any kind of symmetry. This is in contrast with the porous medium equation. For PME not all self-similar solutions are known either rigrously or computationally. However all self-similar solutions whose existence has been proven analytically or by numerical computation have some kind of symmetry. This poses the following

Open problem: which of the self-similar solutions $S_{F}$ of the eikonal equation arise as limits of self-similar solutions of PME as $m \searrow 1$ ? 
Proof of Lemma 1. We apply the Lax-Hopf formula to find the viscosity solution $v$ at time $s>0$ with initial datum $S_{F}(\cdot, t)$ :

$$
\begin{aligned}
v(s, x) & =\sup _{y}\left\{S_{F}(t, y)-\frac{|x-y|^{2}}{4 s}\right\} \\
& =\sup _{y}\left\{\sup _{\xi \in F} W_{\xi}(y, t)-\frac{|x-y|^{2}}{4 s}\right\} \\
& =\sup _{\xi \in F}\left\{\sup _{y} W_{\xi}(y, t)-\frac{|x-y|^{2}}{4 s}\right\} \\
& =\sup _{\xi \in F} W_{\xi}(x, t+s) \\
& =S_{F}(x, t+s)
\end{aligned}
$$

where we have used the fact that $W_{\xi}$ itself is a smooth solution to (EE) and hence also a viscosity solution.

\section{Perturbation of Radial Solutions}

In this section we observe that radial focusing is unstable.

Let $U_{1}: \mathbb{R}^{d} \rightarrow \mathbb{R}$ be any given function, and consider the initial data

$$
u^{\varepsilon}(x)=U_{0}(r)+\varepsilon U_{1}(r \theta),
$$

where $x=r \theta, r>0$ and $|\theta|=1$, i.e. $\theta \in S^{d-1}$ is a unit vector.

The viscosity solution to the initial value problem for (EE) with initial datum $u_{0}^{\varepsilon}$ is

$$
u^{\varepsilon}(x, t)=\sup _{y \in \mathbb{R}^{d}} F(x, t ; y, \varepsilon),
$$

where

$$
F(x, t ; y, \varepsilon)=U_{0}(|y|)+\varepsilon U_{1}(y)-\frac{|x-y|^{2}}{4 t} .
$$

Set $x=\varepsilon \xi, t=T_{*}+\varepsilon \tau$, and $y=\rho \omega$ for $\omega \in S^{d-1}$, and maximize first over $\rho>0$ keeping $\omega$ fixed. For $\varepsilon=0$ the function to be maximized $\rho \mapsto F\left(0, T_{*} ; \rho \omega, 0\right)$ has a unique nondegenerate maximum at $\rho=r_{0}$. since this maximum describes focusing we have

$$
F\left(0, T_{*} ; r_{0} \omega, 0\right)=u^{\varepsilon}\left(0, T_{*}\right)=0 .
$$

and

$$
\omega \cdot F_{y}\left(0, T_{*} ; r_{0} \omega, 0\right)=\left.\frac{\partial}{\partial \rho} F\left(0, T_{*} ; \rho \omega, 0\right)\right|_{\rho=r_{0}}=0 .
$$

By the Implicit Function Theorem $\rho \mapsto F(x, t ; \rho \omega, \varepsilon)$ will have a nondegenerate maximum at $\rho=r_{\varepsilon}(x, t)$ where $r_{\varepsilon}(x, t)$ depends smoothly on $\varepsilon, x$ and $t$. We now compute

$$
\frac{1}{\varepsilon} u^{\varepsilon}(x, t)=\max _{|\omega|=1} \varepsilon^{-1} F\left(x, t ; r_{\varepsilon}(x, t) \omega, \varepsilon\right)
$$


by expanding $F$ in a Taylor series. At the maximizing $\omega$ we have $F_{y}=0$, so we get

$$
\begin{aligned}
F(x, t, ; & \left.r_{\varepsilon}(x, t) \omega, \varepsilon\right) \\
& =F\left(\varepsilon \xi, T_{*}+\varepsilon \tau ;\left(r_{0}+O(\varepsilon)\right) \omega, \varepsilon\right) \\
& =\varepsilon\left\{\xi \cdot F_{x}\left(0, T_{*} ; r_{0} \omega, 0\right)+\tau F_{t}\left(0, T_{*} ; r_{0} \omega, 0\right)+F_{\varepsilon}\left(0, T_{*} ; r_{0} \omega, 0\right)\right\}+O\left(\varepsilon^{2}\right)
\end{aligned}
$$

Computing the relevant derivatives of $F$ and using (9) we get finally,

$$
\frac{1}{\varepsilon} u^{\varepsilon}(x, t)=\max _{|\omega|=1}\left\{U_{1}\left(r_{0} \omega\right)+\frac{r_{0}}{2 T_{*}} \xi \cdot \omega\right\}+\frac{r_{0}^{2}}{4 T_{*}^{2}} \tau+O(\varepsilon) .
$$

so that

$$
\lim _{\varepsilon \rightarrow 0} \frac{1}{\varepsilon} u^{\varepsilon}(x, t)=c P(\xi)+c^{2} \tau,
$$

in which

$$
P(\xi)=\max _{|\omega|=1} \frac{1}{c} U_{1}\left(r_{0} \omega\right)+\xi \cdot \omega
$$

and

$$
c=\frac{r_{0}}{2 T_{*}} .
$$

At time $\tau<0$ the zeroset of the limiting solution in (11) is given by

$$
K_{\tau}=\bigcap_{|\omega|=1}\left\{\xi \mid \xi \cdot \omega \leq-c \tau-\frac{1}{c} U_{1}\left(r_{0} \omega\right)\right\} .
$$

For sufficently large $\tau<0$ we see that $K_{\tau}$ is a convex set with smooth boundary, whose support function is $p(\tau, \omega)=-c \tau-\frac{1}{c} U_{1}\left(r_{0} \omega\right)$. This support function decreases with constant rate $\partial_{\tau} p=-c$, so $\partial K_{\tau}$ shrinks with constant speed $c$. At some moment $\partial K_{\tau}$ will develop a singularity, and after that $K_{\tau}$ still shrinks with constant velocity in the sense of viscosity solutions.

Note that the support function $-c-c^{-1} U_{1}\left(r_{0} \omega\right)$ of $\partial K_{-1}$ can be arbitrary, so that the limiting shape of the shrinking hole can be any convex front moving by Huygens' principle.

\section{Generic Solutions and the Method of Characteristics}

We briefly recall the method of characteristics. In this approach to the solution of (EE) one allows the solution $u(x, t)$ to become multiply valued and considers the graph of this solution together with its space-time gradient, i.e.

$$
\Lambda_{u}=\left\{\left(x, t, u(x, t), \nabla u(x, t), u_{t}(x, t)\right) \mid(x, t) \in \Omega_{t} \times[0, \infty)\right\}
$$

where $\Omega_{t} \subset \mathbb{R}^{d}$ is the domain of the possibly multiply valued function $x \mapsto u(x, t)$. In the language of geometric optics, one calls $\Lambda_{u}$ a front.

This graph $\Lambda_{u}$ is a subset of the 1 -jet space

$$
J^{1}\left(\mathbb{R}^{d+1}\right)=\left\{(x, t, u, p, \dot{u}) \mid x \in \mathbb{R}^{d}, t \in \mathbb{R}, u \in \mathbb{R}, p \in \mathbb{R}^{d}, \dot{u} \in \mathbb{R}\right\}
$$

of $\mathbb{R}^{d+1}$. Points in $J^{1}\left(\mathbb{R}^{d+1}\right)$ are called 1 -jets, or also contact elements.

On the space of contact elements one has the contact form

$$
\theta=d u-p_{1} d x_{1}-\cdots-p_{d} d x_{d}-\dot{u} d t .
$$


$\Lambda_{u}$ is a $d+1$ dimensional submanifold of $J^{1}\left(\mathbb{R}^{d+1}\right)$, and it is even an integral manifold for $\theta$.

The initial datum we prescribe is a smooth function $u_{0}: \Omega_{0} \rightarrow \mathbb{R}$, on some bounded domain $\Omega_{0} \subset \mathbb{R}^{d}$ with smooth boundary $\partial \Omega_{0}$, such that $\nabla u_{0} \neq 0$ on $\partial \Omega_{0}$, while $u_{0}(x)>0$ on $\Omega_{0}$. The front $\Lambda_{u}$ is determined by the initial data through the characteristic flow: namely,

(i) $\Lambda_{u}$ contains the initial surface

$$
\Lambda_{u_{0}}=\left\{\left(x, 0, u_{0}(x), \nabla u_{0}(x), \dot{u}_{0}(x)\right) \mid x \in \mathbb{R}^{d}\right\},
$$

where $\dot{u}_{0}(x)=\left|\nabla u_{0}(x)\right|^{2}$ is the initial velocity, and

(ii) $\Lambda_{u}$ is invariant under the characteristic flow. For (EE) this flow is given by

$$
\left\{\begin{array}{l}
\frac{d x_{k}}{d \tau}=2 p_{k}, \quad \frac{d t}{d \tau}=1, \quad \frac{d p_{k}}{d \tau}=0, \\
\frac{d u}{d \tau}=-\left\{p_{1}^{2}+\cdots+p_{d}^{2}\right\}, \quad \frac{d \dot{u}}{d \tau}=0
\end{array}\right.
$$

( $\tau$ is the time variable for the flow.)

These equations are easily integrated, and they lead to the following parametrization of $\Lambda_{u}$

$$
\left\{\begin{array}{c}
X(t, y)=y-2 t \nabla u_{0}(y) \\
U(t, y)=u_{0}(y)-t\left|\nabla u_{0}(y)\right|^{2} \\
P(t, y)=\nabla u_{0}(y)
\end{array}\right.
$$

LEMMA 2. The map from $\Omega_{0} \times[0, \infty)$ to $J^{1}\left(\mathbb{R}^{d+1}\right)$ given by

$$
(y, t) \mapsto(X(t, y), t, U(t, y), P(t, y), \dot{U}(t, y))
$$

with $\dot{U}(y)=\left|\nabla u_{0}(y)\right|^{2}$ is a Legendre embedding.

Proof. The map $(X, t, U, P) \mapsto(t, y)=(t, X+2 t P)$ is a differentiable inverse for our given map, which shows that it is an embedding.

To verify that it is a Legendre embedding one must show that $d U-P \cdot d X-\dot{U} d t=0$. This can be done by a short direct computation, but it is also guaranteed by the method of characteristics.

Thus $\Lambda_{u}$ is in particular a smooth submanifold of $J^{1}\left(\mathbb{R}^{d+1}\right)$.

If one only looks at the graph of the solution $u$, rather than of $\left(u, \nabla u, u_{t}\right)$, then one finds the following set

$$
\Gamma=\left\{(X(t, y), t, U(t, y)) \mid y \in \Omega_{0}, t \geq 0\right\}
$$

This is a submanifold of $\mathbb{R}^{d} \times[0, \infty) \times \mathbb{R}$ which generally has singularities. The map

$$
(t, y) \mapsto(X(t, y), t, U(t, y))
$$

is called a Legendre mapping (see [7].) 
If one extends the initial data $u_{0}$ by setting $u_{0}(x)=0$ for $x \notin \Omega_{0}$, then the Lax-Hopf formula (1) provides us with a viscosity solution $u^{*}(x, t)$. One can recover $u^{*}$ from $\Gamma$ by considering the upper envelope of $\Gamma$,

$$
u^{*}(x, t)=\max _{(x, t, u) \in \Gamma} u=\left\{\begin{array}{cl}
\max _{X(t, y)=x} U(t, y) & \text { if } \exists_{y \in \Omega_{0}} X(t, y)=x \\
0 & \text { otherwise }
\end{array}\right.
$$

This follows from the Lax-Hopf formula (1): if $y \in \mathbb{R}^{d}$ maximizes $u_{0}(y)-|x-y|^{2} / 4 t$, then, since the viscosity solution is nonnegative one has $u_{0}(y) \geq|x-y|^{2} / 4 t$. Thus either $u_{0}(y)>0$ and hence $y \in \Omega_{0}$, or $u_{0}(y)=0, x=y$ and $u^{*}(x, t)=0$. If $y \in \Omega_{0}$, then the fact that $u_{0}(y)-|x-y|^{2} / 4 t$ has a maximum at $y$ implies $x=y-2 t \nabla u_{0}(y)=X(t, y)$ and $u(x, t)=U(t, y)$.

The singularities of Legendre mappings in general have been studied by the Arnol'd school [7]. The singularities which arise in generic solutions to (EE) and more general Hamilton-Jacobi equations, have been studied by I.A.Bogaevski $[\mathbf{1 2}, \mathbf{1 3}, \mathbf{1 4}]$. He presents a classification the possible singularities by their topological type, at least in dimensions $d=2$ and 3 (the case $d=1$ is classical.) In higher dimensions the classification seems to be very complicated, and even unknown.

Before going on to the free boundary of a solution, we briefly recall some of the singularities found by Bogaevski.

For $d=1$ a generic choice of initial data $u_{0}$ (i.e. for $u_{0}$ in an open and dense subset in the class of $C^{\infty}$ functions) the viscosity solution $u^{*}$ will only have the following singularities:

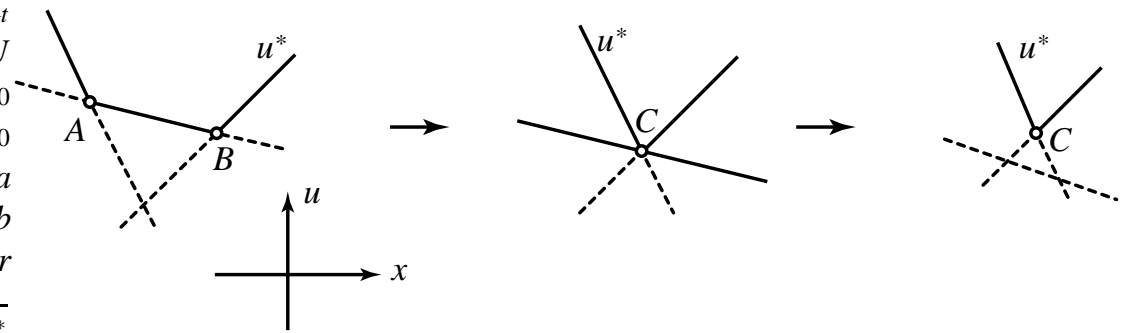

FIgURE 3. Two corners $A$ and $B$ merge into one new corner $C \quad(d=1)$

Except at a finite number of times the viscosity solution $u^{*}$ has only simple corners, which come from transverse self-intersections of the Legendre map defined in (14). At isolated moments in time one such corner can be created in a so-called swallow tail singularity (see figure 4), or two corners can meet and combine to form one new corner (figure 3.)

The more complicated singularities which can only occur at isolated instances in time (swallowtail, and merging corners) have been called perestroikas by the Russian school since they describe how one constellation of simpler singularities can transform into another. 


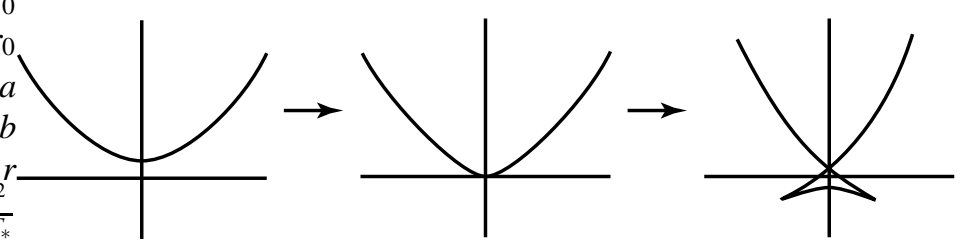

FIGURE 4. Birth of a corner through a swallowtail perestroika $(d=1)$

By combining the curves in figure 4 into one surface one obtains the set $\Gamma$ (figure 5.) The graph of the viscosity solution is obtained by removing the curved tetrahedron $A B C D$ which is "at the bottom" of the surface $\Gamma$.

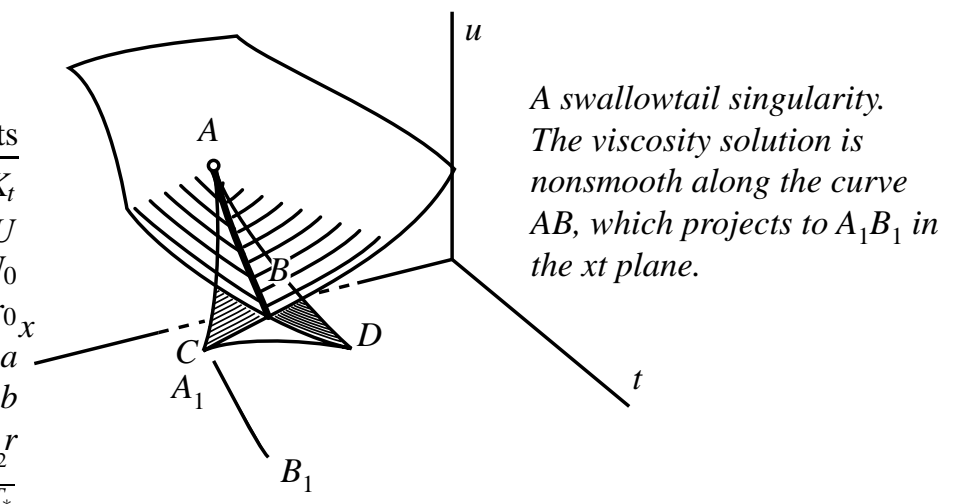

FIGURE 5. A Typical Swallowtail Singularity

\section{Generic Hole Filling}

We now consider the free boundary of a solution. The free boundary of the viscosity solution is the graph of the filling time $T_{*}(x)$ defined in the introduction (see (2).) The filling time is, in general, not a smooth function. To analyze its singularities we use the method of characteristics which shows us that the free boundary is a subset of a Legendre map, and we find that (for generic initial data $u_{0} \in C^{\infty}\left(\bar{\Omega}_{0}\right)$ ) its singularities are those of a generic Legendre map. The generic singularities of the filling time are then those obtained by Bogaevski for generic minimum functions [12].

The free boundary is given by setting $U(t, y)=0$. This leads to an equation $\left(u_{0}(y)-\right.$ $\left.t\left|\nabla u_{0}(y)\right|^{2}=0\right)$ for the time $t$ it takes the contact element $\left(y, 0, u_{0}(y), \nabla u_{0}(y), \dot{u}_{0}(y)\right)$ to reach the level $\{u=0\}$ under the characteristic flow. Let

$$
\Theta(y)=u_{0}(y) /\left|\nabla u_{0}(y)\right|^{2}
$$

be this time. When the contact element reaches $\{u=0\}$, it finds itself at $x=X(y)$, where

$$
X(y)=y-\frac{2 u_{0}(y)}{\left|\nabla u_{0}(y)\right|^{2}} \nabla u_{0}(y)
$$


The map $y \mapsto(X(y), \Theta(y))$ then parametrizes the extended free boundary (extended, because it will also include those points where $\Gamma$ hits the set $u=0$.) In general $\Theta$ is not single valued, and the map $y \mapsto(X(y), \Theta(y))$ will have singularities. One can again analyze these by considering the graph of $\Theta$ and its gradient. This graph is contained in the 1 -jet space $J^{1}\left(\mathbb{R}^{d}\right)$, it is parametrized by

$$
\Theta(y)=\frac{u_{0}(y)}{\left|\nabla u_{0}(y)\right|^{2}}, \quad N(y)=-\frac{\nabla u_{0}(y)}{\left|\nabla u_{0}(y)\right|^{2}} .
$$

LEMMA 3. Let $\Omega_{0}^{*}=\left\{y \in \Omega_{0} \mid \nabla u_{0}(y) \neq 0\right\}$. The map $\Phi$ from $\Omega_{0}^{*}$ into $J^{1}\left(\mathbb{R}^{d}\right)$ given by

$$
\Phi: y \mapsto(X(y), \Theta(y), N(y))
$$

is a smooth, proper Legendre embedding.

PROOF. A smooth inverse is obtained by direct computation,

$$
\nabla u_{0}=-\frac{N}{|N|^{2}} \Rightarrow u_{0}=\Theta\left|\nabla u_{0}\right|^{2}=\Theta|N|^{-2} \Rightarrow y=X+\frac{2 u_{0}}{\left|\nabla u_{0}\right|^{2}} \nabla u_{0}=X+\frac{2 \Theta}{|N|^{2}} N
$$

So the map is a smooth embedding.

As $y$ approaches the zeroset of $\nabla u_{0}(y)$ the quantity $\Theta(y)$ becomes unbounded, so the map $\Phi$ is proper.

To verify that $\Phi$ is a Legendre embedding one must show $d \Theta-N \cdot d X=0$. This can be done by an straightforward computation in which one verifies that $\partial \Theta / \partial y_{j}=N \cdot\left(\partial X / \partial y_{j}\right)$ for $j=1, \ldots, d$. Alternatively one can deduce this from the fact that $\Phi$ parametrizes the Legendre submanifold of $J^{1}\left(\mathbb{R}^{d}\right)$ obtained by slicing $\Lambda_{u}$ with $\{u=0\}$.

The following (easy) observation is the key to the analysis of generic singularities of the free boundary.

LEMMA 4. Let $u_{0} \in C^{\infty}\left(\bar{\Omega}_{0}\right)$ be given, and let $\Phi_{0}$ be its corresponding generalized free boundary. Then any sufficiently small perturbation of $\Phi_{0}$ with in the class of $C^{\infty}$ Legendre immersions can be achieved by an appropriately chosen small perturbation of the initial data $u_{0} \in C^{\infty}\left(\bar{\Omega}_{0}\right)$.

We use the characteristic flow to solve the inverse problem in which one determines the initial data from a given free boundary. This is of course only possible for the generalized solution in the sense of Legendre submanifolds: For viscosity solutions the free boundary does not determine the initial data uniquely.

PROOF. Let $\tilde{\Lambda} \subset J^{1}\left(\mathbb{R}^{d}\right)$ be any Legendre submanifold (such as the extended free boundary, i.e. the image of $\Phi)$. We embed this Legendre submanifold in $J^{1}\left(\mathbb{R}^{d+1}\right)$ by mapping the contact element $(X, \Theta, N) \in \tilde{\Lambda} \subset J^{1}\left(\mathbb{R}^{d}\right)$ to the contact element

$$
(x, t, u, p, \dot{u})=\left(X, \Theta, 0,-\frac{N}{|N|^{2}}, \frac{1}{|N|^{2}}\right) \in J^{1}\left(\mathbb{R}^{d+1}\right) .
$$

The union of all characteristics passing throught the points in $J^{1}\left(\mathbb{R}^{d+1}\right)$ thus obtained gives us a Legendre submanifold of $J^{1}\left(\mathbb{R}^{d+1}\right)$ which satisfies $\dot{u}=|p|^{2}$. Slicing this large Legendre submanifold with the section $\{t=0\}$ then gives a Legendre submanifold $\Sigma \subset$ 
$J^{1}\left(\mathbb{R}^{d}\right)$. If this last Legendre submanifold $\Sigma$ is the graph of some smooth function $u_{1}$ and its gradient $\nabla u_{1}$, then $u_{1}$ is the initial function whose free boundary is $\tilde{\Lambda}$.

In our situation $\tilde{\Lambda}$ is a small perturbation of the free boundary of the solution with initial function $u_{0}$. In this case the initial Legendre submanifold $\Sigma \subset J^{1}\left(\mathbb{R}^{d}\right)$ corresponding to the perturbed free boundary $\tilde{\Lambda}$ will be a small perturbation of the graph of $\left(u_{0}, \nabla u_{0}\right)$. Hence $\Sigma$ will also be a graph of some function $x \mapsto\left(u_{1}(x), p(x)\right)$. The Legendre condition forces $p(x)=\nabla u_{1}(x)$.

From this lemma we see that the filling time is just a generic minimum function, i.e. generic choices of $u_{0}$ lead to functions with the same kind of singularities as functions of the type

$$
T(x)=\min _{y \in \Omega_{0}} F(x, y)
$$

in which $F: \Omega_{0} \times \Omega_{0} \rightarrow \mathbb{R}$ is a generically chosen smooth function of two variables.

Close scrutiny of Bogaevski's list of possible singularities of minimum functions reveals that there are only two possible ways in which a hole can be filled when $d=2$, namely, the vanishing triangle and the closing eye.

The "vanishing triangle" is locally described to leading order by

$$
u(x, t)=\max \left\{0, w_{1}(x, t), w_{2}(x, t), w_{3}(x, t)\right\}
$$

where the $w_{j}$ are three plane waves converging upon the focusing point at time $t=T$. It is thus an asymptotically self-similar solution. In the language of singularity theory this

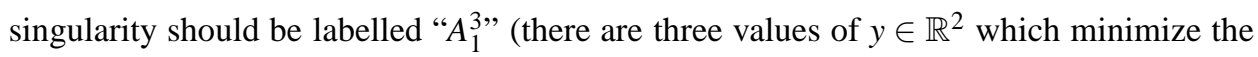
quantity $Q^{x}(y)=|x-y|^{2} / 4 u_{0}(y)$ in the definition of the filling time, and these three minima each are nondegenerate critical points of $Q^{x}$.)

The "closing eye" is locally described by

$$
u(x, t)=\max \left\{0, q_{+}(x, t), q_{-}(x, t)\right\}
$$

where near $(x, y, t)=(0,0,0)$ one has

$$
q_{ \pm}(x, t)= \pm\left\{y-c_{ \pm} x^{2}+\left(1+4 c_{ \pm}^{2} x^{2}\right) t x^{2}+\cdots\right\} .
$$

In the language of singularity theory this singularity should be labelled " $A_{1}^{2}$ " (there are two values of $y \in \mathbb{R}^{2}$ which minimize the quantity $Q^{x}(y)=|x-y|^{2} / 4 u_{0}(y)$, and these two minima each are nondegenerate critical points of $Q^{x}$.) 


\section{References}

[1] S.B.Angenent, D.G.Aronson, The focussing problem for the readially symmetric porous medium equation, Comm. P.D.E. 20(1995), 1217-1240.

[2] S.B.Angenent, D.G.Aronson, Non-axial self-similar hole filling for the porous medium equation,J.A.M.S. 14(2001), 737-782.

[3] S.B.Angenent, D.G.Aronson, S.Betelú, J.S.Lowengrub, Focusing of an elongated hole in porous medium flow, Physica D 151 (2001), no. 2-4, 228-252.

[4] V.I.Arnol'd, Geometrical methods in the Theory of Ordinary Differential Equations, Springer Grundlehren, 250, (1983).

[5] V.I.Arnol'd, Catastrophe Theory, Springer Verlag, 2nd edition, (1984).

[6] V.I.Arnol'd, Mathematical Methods of Classical Mechanics, Graduate Texts in Mathematics, 60 Springer-Verlag, New York, 1991.

[7] V.I.Arnol'd, Singularities of Caustics And WaVe Fronts, Mathematics and its Applications - Soviet series 62, Kluwer 1990.

[8] D.G. Aronson, The porous medium equation, in: Some Problems in Nonlinear Diffusion (A. Fasano and M. Primicerio edditors), Lecture Notes on Math. 1224, Springer, Berlin 1986.

[9] D.G. Aronson and J. Graveleau, A self-similar solution to the focusing problem for the porous medium equation, Euro. J. Appl. Math. 4 (1993), 65-81.

[10] G.I. Barenblatt, Similarity, SElF-Similarity AND InTERmediate ASYMPtotics, Consultants Bureau, New York, 1978.

[11] S.I.Betelú, D.G. Aronson, S.B. Angenent, Renormalization study of two-dimensional convergent solutions of the porous medium equation, Phys. D 138 (2000), no. 3-4, 344-359.

[12] I.A.Bogaevski, Reconstructions of singularities of minimum functions, and bifurcations of shock waves of the Burgers equation with vanishing viscosity, (Russian) Algebra i Analiz 1 (1989), 4, 1-16; translation in Leningrad Math. J. 1 (1990), 4, 807-823

[13] I.A.Bogaevskiĭ, Perestroikas of fronts in evolutionary families, (Russian) Trudy Mat. Inst. Steklov. 209 (1995), Osob. Gladkikh Otobrazh. s Dop. Strukt., 65-83.

[14] I.A.Bogaevski, Singularities of viscosity solutions of Hamilton-Jacobi equations, Singularity theory and differential equations (Kyoto, 1999). Sūrikaisekikenkyūsho Kōkyūroku 1111 (1999), 138-143.

[15] L.C.Evans, Partial Differential Equations, Graduate Studies in Mathematics 19, A.M.S. 1998.

[16] F.John, Partial Differential Equations, Applied Mathematical Sciences 1 4th edition, Springer Verlag, 1982.

[17] P.-L.Lions, P.E.Souganidis, J.L. Vázquez, The relation between the porous medium and the eikonal equations in several space dimensions, Rev. Mat. Iberoamericana 3 (1987), no. 3-4, 275-310.

Mathematics Department, UW Madison, Wisconsin

E-mail address: angenent@math.wisc.edu

School of Mathematics, University of Minnesota, Minneapolis

E-mail address: don@math.umn.edu 